\title{
Analytical study of the proximity of exoplanetary systems to mean-motion resonances
}

\begin{abstract}
A.-S. Libert ${ }^{\star}$ and J. Henrard
Département de mathématique FUNDP, 8 Rempart de la Vierge, 5000 Namur, Belgium

e-mail: anne-sophie.libert@fundp.ac.be

Received 7 June 2006 / Accepted 11 September 2006

ABSTRACT

Aims. In a previous paper (Libert \& Henrard 2005, Celest. Mech. \& Dyn. Astron., 93, 187) we used a twelfth-order expansion of the perturbative potential in powers of eccentricities to represent the secular effects of two coplanar planets. This expansion was applied successfully to non resonant exoplanetary systems. This study was based on a first order (in the masses of the planets) model and will fail for systems too close to a resonance. In this paper we test the effects of the proximity of a mean-motion resonance on the secular motion of the planets.

Methods. We analyse the proximity of several exoplanetary systems to a mean-motion resonance zone by using a first-order (in the mass ratios) Lie algorithm on the perturbative potential expanded to the twelfth order in the eccentricities. This perturbation method evaluates the difference between osculating elements and averaged ones. It permits us to decide whether resonant contributions dominate the terms of this difference or not.

Results. This study is applied to several exosystems. We find that HD 168443, HD 38529, HD 74156, HD 217107, and HD 190360 are far away from a mean-motion resonance zone. $v$ Andromedae and HD 12661 are rather close to the 5/1 resonance, HD 169830 to the $9 / 1$ one. Hence, a secular theory is enough to depict correctly the behaviour of all these systems. On the other hand, HD 108874 and HD 202206 suffer from large perturbations in their motion due to the closeness of the 4/1 and 5/1 resonances, respectively. We also perform a complete investigation of the proximity of the $v$ Andromedae system to mean-motion resonances, by studying the changes in behaviour due to different values of the outer semi-major axis. The $v$ Andromedae system begins to be really influenced by the $5 / 1$ resonant terms when the value of the outer semi-major axis decreases from 2.53 to 2.445 .
\end{abstract}

Key words. celestial mechanics - planetary systems - methods: analytical

\section{Introduction}

At present more than 18 multiple exoplanetary systems have been discovered. Some of them are in mean-motion resonances, for example GJ876 and HD 82943 in resonance 2/1. This firstorder resonance has been studied by several authors (Beaugé et al. 2006; Psychoyos \& Hadjidemetriou 2005, etc.). Looking at the period ratio of the other two-planet systems discovered so far (see the orbital parameters listed in Table 1), it seems that most of them are not close to mean-motion resonances so that their behaviour can be explained by their mutual secular interactions alone. The secular dynamics of some of these have already been studied in several papers. We may cite for instance the works of Chiang et al. (2001), Ji et al. (2003), Michtchenko \& Malhotra (2004), and Lee \& Peale (2003).

In a previous paper (Libert \& Henrard 2005), we showed that an analytical theory based on a high-order expansion of the secular Hamiltonian in powers of eccentricities could correctly depict the motion of these exosystems. In order to obtain the expansion of the secular perturbation, we simply discarded the terms that depend upon the mean anomalies of the planets. This amounts to an "averaging by scissors", or a first-order (in the mass ratios) averaging of the non secular terms of the perturbation. Of course, this averaging will fail inside the mean-motion resonance, but also will be a bad approximation if the system is close to the resonance. This is well-known in the solar system,

\footnotetext{
* FNRS Research Fellow.
}

where Jupiter and Saturn are close to a 5 to 2 resonance, leading to large perturbations in their motion, the so-called "great inequality". The neighbourhood of this resonance region has been studied by Michtchenko \& Ferraz-Mello (2001) among others. The same kind of work has been performed by Callegari et al. (2004) for the Uranus-Neptune system.

In order to test the possibility of "great inequalities" in the exosystems ( $v$ Andromedae, HD 168443, HD 169830, HD 38529, HD 74156, and HD 12661) that we studied earlier and new ones like HD 108874, HD 202206, HD 217107, and HD 190360, we implement a complete first-order (in the mass of the planets) perturbation method. For a system very close to a resonance such that $k_{1} n_{1}-k_{2} n_{2}=0$, the solution is dominated by terms that include a combination of " $k_{1} \lambda_{1}-k_{2} \lambda_{2}$ "; the amplitudes of these terms give a measure of the closeness to the resonance. For systems farther away from the resonance, short periodic terms dominate. The dominating terms (tabulated in Table 2) also measure the difference between osculating elements and averaged elements, giving an idea of what resonant and non resonant terms influence significantly the motion.

\section{Effects of the closeness of exoplanetary systems to mean-motion resonances}

\subsection{First-order Lie averaging}

Let us consider a system consisting of a central star of mass $m_{0}$ and two coplanar planets of mass $m_{1}$ and $m_{2}$. Mass $m_{1}$ is 
Table 1. Orbital parameters of some exoplanetary systems released on the web site www . obspm. fr/encycl/encycl.html (March 2006).

\begin{tabular}{lcccccccc}
\hline \hline & $a_{1}$ & $a_{2}$ & $e_{1}$ & $e_{2}$ & $\varpi_{1}(\mathrm{deg})$ & $\varpi_{2}(\mathrm{deg})$ & $m_{1} / M_{J}$ & $m_{2} / M_{J}$ \\
\hline$v$ Andro (c-d) & 0.829 & 2.53 & 0.28 & 0.27 & 250 & 260 & 1.89 & 3.75 \\
HD 168443 & 0.29 & 2.87 & 0.529 & 0.228 & 172.9 & 63 & 7.2 & 17.1 \\
HD 169830 & 0.81 & 3.6 & 0.31 & 0.33 & 148 & 252 & 2.88 & 4.04 \\
HD 38529 & 0.129 & 3.68 & 0.29 & 0.36 & 87.7 & 14.7 & 0.78 & 12.7 \\
HD 74156 & 0.294 & 3.4 & 0.636 & 0.583 & 181.5 & 242.4 & 1.86 & 6.17 \\
HD 12661 & 0.83 & 2.56 & 0.35 & 0.2 & 291.73 & 162.4 & 2.3 & 1.57 \\
HD 108874 & 1.051 & 2.68 & 0.07 & 0.25 & 248.4 & 17.3 & 1.36 & 1.018 \\
HD 202206 & 0.83 & 2.55 & 0.435 & 0.267 & 161.18 & 78.99 & 17.4 & 2.44 \\
HD 217107 & 0.074 & 4.3 & 0.13 & 0.55 & 21.1 & 164 & 1.37 & 2.1 \\
HD 190360 & 0.128 & 3.92 & 0.01 & 0.36 & 153.7 & 153.7 & 0.057 & 1.502 \\
\hline
\end{tabular}

assumed to be the one closest to the central star. The Hamiltonian of the dynamics of this system, in the usual Jacobi coordinates and limited to the first order in the mass ratios $m_{1} / m_{0}$ and $m_{2} / m_{0}$, is:

$\mathcal{H}=-\frac{G m_{0} m_{1}}{2 a_{1}}-\frac{G m_{0} m_{2}}{2 a_{2}}-G m_{1} m_{2}\left[\frac{1}{\left|\boldsymbol{r}_{1}-\boldsymbol{r}_{2}\right|}-\frac{\left(\boldsymbol{r}_{1} \mid \boldsymbol{r}_{2}\right)}{r_{2}^{3}}\right]$,

where $a_{i}, \boldsymbol{r}_{i}$, and $r_{i}$ are, respectively, the osculating semi-major axis, the position vector, and the norm of the position vector of mass $m_{i}$ (see for instance Brouwer \& Clemence 1961).

Using the classical modified Delaunay's elements (truncated at the first order in the mass ratios) that are (see reference above or Laskar 1990)

$\lambda_{i}=$ mean longitude of $m_{i}, \quad L_{i}=m_{i} \sqrt{G m_{0} a_{i}}$,

$p_{i}=-$ the longitude of the pericenter of $m_{i}$,

$P_{i}=L_{i}\left[1-\sqrt{1-e_{i}^{2}}\right]$,

the last term of Eq. (1) can be expanded in powers of the eccentricities (see for instance Murray \& Dermott 1999). Considering the expressions $E_{i}=\sqrt{2 P_{i} / L_{i}}$ (we call them "canonical" eccentricities because they are closely related to the action $P_{i}$ ) rather than the eccentricities $e_{i}$ (for small to moderate eccentricities $E_{i}$ is close to $e_{i}$ ), the Hamiltonian reads

$$
\begin{aligned}
\mathcal{H}= & -\frac{G m_{0} m_{1}}{2 a_{1}}-\frac{G m_{0} m_{2}}{2 a_{2}} \\
& -\frac{G m_{1} m_{2}}{a_{2}} \sum B_{i_{1}, i_{2}, j_{1}, j_{2}}^{k} E_{1}^{\left|j_{1}\right|+2 i_{1}} E_{2}^{\left|j_{2}\right|+2 i_{2}} \cos \Phi,
\end{aligned}
$$

where $\Phi=\left[\left(k+j_{1}\right) \lambda_{1}-\left(k+j_{2}\right) \lambda_{2}+j_{1} p_{1}-j_{2} p_{2}\right]$ and the sum runs on all values of the integer indices $\left(k, i_{1}, i_{2}, j_{1}, j_{2}\right)$ (the first three being positive). The coefficients $B_{i_{1}, i_{2}, j_{1}, j_{2}}^{k}$ depend only on the ratio $a_{1} / a_{2}$ of the semi-major axes.

As shown in our previous paper (Libert \& Henrard 2005), the numerical convergence of the secular part of the series (2) (those terms for which $j_{1}=j_{2}=-k$ ) is excellent for the exosystems under consideration. Some readers may wonder if this does not contradict the results of Henrard \& Lemaître (1987) and Lemaître \& Henrard (1988), but we do want to point out a basic difference: we investigate the (numerical) convergence of the secular part only and not of the full expansion. Despite their high eccentricities, the exosystems of Table 1 enjoy a good convergence and, if we limit the development to order 12, the expansion of the secular part can represent the orbits with enough accuracy. For more information on this analytical model we refer to our previous articles (Libert \& Henrard 2005, 2006).

To obtain the expansion of the secular perturbation, we simply dropped the terms depending upon the mean anomalies of the planets from the Hamiltonian (2). This amounts to an "averaging by scissors", or a first-order (in the mass ratios) averaging of the non secular terms of the perturbation. In this paper we perform a complete first-order Lie transform (Hori 1966; Deprit 1969 ) instead. It again yields the secular Hamiltonian but also a first-order generator necessary for the calculation of the averaged initial elements:

$$
W_{1}=-\frac{G m_{1} m_{2}}{a_{2}} \sum \frac{B_{i_{1}, i_{2}, j_{1}, j_{2}}^{k} E_{1}^{\left|j_{1}\right|+2 i_{1}} E_{2}^{\left|j_{2}\right|+2 i_{2}}}{\left(k+j_{1}\right) n_{1}-\left(k+j_{2}\right) n_{2}} \sin \Phi,
$$

where the $n_{i}=\sqrt{G m_{0} / a_{i}^{3}}$ expresses the Keplerian mean-motion of the planets.

Notice that we can substitute the non-constant meanmotions $n_{i}$ for the constant averaged values in the denominators. The error committed is of the second order in the masses. The generator $W_{1}$ enables us to define the effects of the perturbation to the first order (in the masses). For instance, we easily compute (with $\epsilon=m_{1} m_{2} / m_{0}^{2}$ ):

$E_{i}^{2}=\bar{E}_{i}^{2}+\epsilon\left[E_{i}^{2} ; W_{1}\right]+O\left(\epsilon^{2}\right)$

where the expression $\bar{E}_{i}$ designates averaged values and the notation [;] stands for the Poisson bracket.

Indeed, $E_{i}^{2}=2 P_{i} / L_{i}$ and $\left[E_{i}^{2} ; W_{1}\right]=\left[E_{i}^{2}\left(\partial W_{1} / \partial \lambda_{i}\right)-\right.$ $\left.2\left(\partial W_{1} / \partial p_{i}\right)\right] / L_{i}$. Notice that in spite of the fact that $\epsilon$ is of order 2 in the masses of the planets, the correcting term is actually of order 1 because the denominator $L_{i}$ is also of order 1 in the masses.

Taking the square root of this expression and applying a firstorder Taylor expansion, we find

$E_{i}=\bar{E}_{i}\left(1+\frac{\epsilon}{2 E_{i}^{2}}\left[E_{i}^{2} ; W_{1}\right]\right)+O\left(\epsilon^{2}\right)$.

The higher the terms generated by the Poisson bracket, the stronger their influences on the value of the "canonical" eccentricities. In fact the amplitude of a periodic term gives us a measure of the proximity of the system to the corresponding meanmotion resonances, as we will see in the next section.

\subsection{Application to several exoplanetary systems}

This section analyses the closeness of several systems to a mean-motion resonance zone. The orbital parameters of the considered exosystems are released on the web site www.obspm.fr/encycl/encycl.html and listed in Table 1. The $v$ Andromedae system actually has three known planets but 
Table 2. Proximity of several planetary sytems to mean-motion resonances by analysing the largest terms of $\frac{\epsilon}{2 E_{1}^{2}}\left[E_{1}^{2} ; W_{1}\right]$ and $\frac{\epsilon}{2 E_{2}^{2}}\left[E_{2}^{2} ; W_{1}\right]$ : HD $168443\left(a_{1} / a_{2}=0.1\right), \operatorname{HD} 38529\left(a_{1} / a_{2}=0.035\right), \operatorname{HD} 74156\left(a_{1} / a_{2}=0.086\right), v$ Andromedae $\left(a_{1} / a_{2}=0.328\right), \operatorname{HD} 12661\left(a_{1} / a_{2}=0.324\right)$, HD $169830\left(a_{1} / a_{2}=0.225\right)$, HD $108874\left(a_{1} / a_{2}=0.392\right)$, and coplanar Jupiter-Saturn $\left(a_{1} / a_{2}=0.546\right)$.

\begin{tabular}{lrr}
\hline \hline & $\frac{\epsilon}{2 E_{1}^{2}}\left[E_{1}^{2} ; W_{1}\right]$ & $\frac{\epsilon}{2 E_{2}^{2}}\left[E_{2}^{2} ; W_{1}\right]$ \\
\hline HD 168443 & $-0.00192 \cos \left(2 \lambda_{2}+2 p_{1}\right)$ & $-0.0598 \cos \left(\lambda_{2}+p_{2}\right)$ \\
& $-0.00103 \cos \left(3 \lambda_{2}+2 p_{1}+p_{2}\right)$ & $-0.0138 \cos \left(2 \lambda_{2}+2 p_{2}\right)$ \\
\hline HD 38529 & $-0.000214 \cos \left(2 \lambda_{2}+2 p_{1}\right)$ & $-0.00293 \cos \left(\lambda_{2}+p_{2}\right)$ \\
& $-0.000183 \cos \left(3 \lambda_{2}+2 p_{1}+p_{2}\right)$ & $0.00107 \cos \left(2 \lambda_{2}+2 p_{2}\right)$ \\
\hline HD 74156 & $0.00102 \cos \left(8 \lambda_{2}+2 p_{1}+6 p_{2}\right)$ & $-0.00555 \cos \left(\lambda_{2}+p_{2}\right)$ \\
& $0.00101 \cos \left(9 \lambda_{2}+2 p_{1}+7 p_{2}\right)$ & $-0.00341 \cos \left(2 \lambda_{2}+2 p_{2}\right)$ \\
\hline$v$ Andro (c-d) & $0.0121 \cos \left(\lambda_{1}-5 \lambda_{2}-2 p_{1}-2 p_{2}\right)$ & $-0.0111 \cos \left(\lambda_{2}+p_{2}\right)$ \\
& $-0.00998 \cos \left(\lambda_{1}-5 \lambda_{2}-p_{1}-3 p_{2}\right)$ & $-0.00931 \cos \left(\lambda_{1}-5 \lambda_{2}-p_{1}-3 p_{2}\right)$ \\
& $-0.00481 \cos \left(\lambda_{1}-5 \lambda_{2}-3 p_{1}-p_{2}\right)$ & $0.00375 \cos \left(\lambda_{1}-5 \lambda_{2}-2 p_{1}-2 p_{2}\right)$ \\
\hline HD 12661 & $-0.00257 \cos \left(\lambda_{1}-5 \lambda_{2}-2 p_{1}-2 p_{2}\right)$ & $-0.0222 \cos \left(\lambda_{2}+p_{2}\right)$ \\
& $-0.00172 \cos \left(\lambda_{1}-5 \lambda_{2}-3 p_{1}-p_{2}\right)$ & $-0.00987 \cos \left(\lambda_{1}-5 \lambda_{2}-p_{1}-3 p_{2}\right)$ \\
& $-0.00126 \cos \left(\lambda_{1}-5 \lambda_{2}-p_{1}-3 p_{2}\right)$ & $0.0067 \cos \left(\lambda_{1}-5 \lambda_{2}-2 p_{1}-2 p_{2}\right)$ \\
\hline HD 169830 & $0.00183 \cos \left(\lambda_{1}-9 \lambda_{2}-2 p_{1}-6 p_{2}\right)$ & $-0.0122 \cos \left(\lambda_{2}+p_{2}\right)$ \\
& $-0.00144 \cos \left(\lambda_{1}-9 \lambda_{2}-3 p_{1}-5 p_{2}\right)$ & $-0.00417 \cos \left(2 \lambda_{2}+2 p_{2}\right)$ \\
& $-0.00115 \cos \left(2 \lambda_{2}+2 p_{1}\right)$ & $-0.00206 \cos \left(\lambda_{1}-9 \lambda_{2}-p_{1}-7 p_{2}\right)$ \\
\hline HD 108874 & $-0.1337 \cos \left(\lambda_{1}-4 \lambda_{2}-p_{1}-2 p_{2}\right)$ & $-0.0173 \cos \left(\lambda_{1}-4 \lambda_{2}-p_{1}-2 p_{2}\right)$ \\
& $0.0338 \cos \left(\lambda_{1}-4 \lambda_{2}-2 p_{1}-p_{2}\right)$ & $-0.0117 \cos \left(\lambda_{2}+p_{2}\right)$ \\
& $0.0186 \cos \left(\lambda_{1}-4 \lambda_{2}-p_{1}-2 p_{2}\right)$ & $-0.00973 \cos \left(\lambda_{1}-4 \lambda_{2}-3 p_{2}\right)$ \\
\hline Jup-Sat & $-0.0272 \cos \left(\lambda_{1}-2 \lambda_{2}-p_{1}\right)$ & $0.0486 \cos \left(2 \lambda_{1}-5 \lambda_{2}-p_{1}-2 p_{2}\right)$ \\
& $-0.0133 \cos \left(2 \lambda_{1}-5 \lambda_{2}-2 p_{1}-p_{2}\right)$ & $-0.0462 \cos \left(\lambda_{2}+p_{2}\right)$ \\
& $0.0123 \cos \left(2 \lambda_{1}-5 \lambda_{2}-p_{1}-2 p_{2}\right)$ & $-0.0444 \cos \left(2 \lambda_{1}-5 \lambda_{2}-3 p_{2}\right)$ \\
\hline
\end{tabular}

our study only concerns the two outer ones (c-d). Table 2 provides information about the largest coefficients of the expressions $\frac{\epsilon}{2 E_{1}^{2}}\left[E_{1}^{2} ; W_{1}\right]$ and $\frac{\epsilon}{2 E_{2}^{2}}\left[E_{2}^{2} ; W_{1}\right]$ (developed to order 12 in the eccentricities).

First we observe that the exoplanetary systems HD 168443, HD 38529, and HD 74156 from Table 2 are far away from a mean-motion resonance zone, their behaviour being in fact determined by terms that do not include any combination of " $k_{1} \lambda_{1}-$ $k_{2} \lambda_{2}$ ". These systems are also called hierarchical systems because of their small semi-major axes ratio. This is also the case for HD 217107 and HD 190360 of Table 1.

However Table 2 shows that the exosystems $v$ Andromedae and HD 12661 are rather close to the 5/1 resonance. Actually their behaviour is determined this time by terms including a combination of " $\lambda_{1}-5 \lambda_{2}$ ", but the coefficients of these periodic terms remain small (order $10^{-3}$ ). We also see the greater influence of non resonant terms on the outer body than of resonant ones. HD 169830 seems close to the 9/1 resonance, but a secular theory is enough to depict the behaviour of such nearresonant systems correctly, as it is shown by the figures in the next section.

To see the influence of the proximity of mean-motion resonances on the magnitude of the coefficients, we also added the well-known Jupiter-Saturn system, which is here assumed to be coplanar. It is known that these planets suffer from large perturbations in their motion due to the closeness of the $5 / 2$ resonance. The coefficients of the resonant periodic terms are dominant this time and of higher order $\left(10^{-1}\right.$ or $\left.10^{-2}\right)$. The exosystems HD 108874 (close to 4/1 resonance) and HD 20206 of Table 1 (close to 5/1 resonance) tend to show the same behaviour as the Jupiter-Saturn system. For these last three systems, a secular theory is not enough to depict their behaviour correctly, as we see in the next section.

\section{Investigation of the proximity of the $v$ Andromedae system to mean-motion resonances}

As seen in Table 2, the motion of the $v$ Andromedae system is influenced by its proximity to the $5 / 1$ resonance. In order to study the extent of the influence of the $5 / 1$ zone, we vary the ratio of the semi-major axes in this section, keeping the other parameters and initial conditions at the same values.

Table 3 shows the largest terms of $\frac{\epsilon}{2 E_{1}^{2}}\left[E_{1}^{2} ; W_{1}\right]$ and $\frac{\epsilon}{2 E_{2}^{2}}\left[E_{2}^{2} ; W_{1}\right]$ for several values of the outer semi-major axis. All the other parameters (including the inner semi-major axis) are constant and are those of the $v$ Andromedae system. First, if $a_{1} / a_{2}=0.322$, the proximity of the $11 / 2$ resonance (at $a_{1} / a_{2}=$ 0.321 ) has some influence on the results. When $a_{1} / a_{2}=0.328$, it is the resonance $5 / 1$ (at $a_{1} / a_{2}=0.342$ ) that becomes dominant. Figure 1 shows the comparison between our secular analytical approach and the numerical integration of the full threebody problem, using the SWIFT software package developed by Duncan \& Levison (Wisdom-Holman mapping, 1991). But actually we see that for these two first semi-major axis ratios, the dynamics of the system is dominated by non resonant terms, so the secular theory should give a good approximation of their motion.

The same result has been pointed out by Rodríguez \& Gallardo (2005) concerning the system HD 12661. With the orbital parameters obtained by D. Fischer, they show that it was dominated by a purely secular evolution that is reproduced very well with a disturbing function that includes only sixth-order terms in the eccentricities. We may point out that, in evaluating the closeness to resonance, they consider only the size of the combination of frequencies (the denominator of the resonant term), while we also consider the size of the perturbing term (the numerator of the resonant term). For instance, in the case of HD 12661 (with the values of the parameters adopted in Table 1), 
Table 3. Largest terms of $\frac{\epsilon}{2 E_{1}^{2}}\left[E_{1}^{2} ; W_{1}\right]$ and $\frac{\epsilon}{2 E_{2}^{2}}\left[E_{2}^{2} ; W_{1}\right]$ for the $v$ Andromedae system with different outer semi-major axis $a_{2}$.

\begin{tabular}{rrr}
\hline \hline$a_{1} / a_{2}$ & $\frac{\epsilon}{2 E_{1}^{2}}\left[E_{1}^{2} ; W_{1}\right]$ & $\frac{\epsilon}{2 E_{2}^{2}}\left[E_{2}^{2} ; W_{1}\right]$ \\
\hline 0.322 & $0.0127 \cos \left(2 \lambda_{1}-11 \lambda_{2}-3 p_{1}-6 p_{2}\right)$ & $-0.01106 \cos \left(\lambda_{2}+p_{2}\right)$ \\
& $-0.0101 \cos \left(2 \lambda_{1}-11 \lambda_{2}-4 p_{1}-5 p_{2}\right)$ & $-0.00983 \cos \left(2 \lambda_{1}-11 \lambda_{2}-2 p_{1}-7 p_{2}\right)$ \\
& $-0.0091 \cos \left(2 \lambda_{1}-11 \lambda_{2}-2 p_{1}-7 p_{2}\right)$ & $0.00785 \cos \left(2 \lambda_{1}-11 \lambda_{2}-3 p_{1}-6 p_{2}\right)$ \\
\hline 0.328 & $0.0121 \cos \left(\lambda_{1}-5 \lambda_{2}-2 p_{1}-2 p_{2}\right)$ & $-0.0111 \cos \left(\lambda_{2}+p_{2}\right)$ \\
& $-0.00998 \cos \left(\lambda_{1}-5 \lambda_{2}-p_{1}-3 p_{2}\right)$ & $-0.00931 \cos \left(\lambda_{1}-5 \lambda_{2}-p_{1}-3 p_{2}\right)$ \\
& $-0.00481 \cos \left(\lambda_{1}-5 \lambda_{2}-3 p_{1}-p_{2}\right)$ & $0.00375 \cos \left(\lambda_{1}-5 \lambda_{2}-2 p_{1}-2 p_{2}\right)$ \\
\hline 0.339 & $0.0673 \cos \left(\lambda_{1}-5 \lambda_{2}-2 p_{1}-2 p_{2}\right)$ & $-0.0511 \cos \left(\lambda_{1}-5 \lambda_{2}-p_{1}-3 p_{2}\right)$ \\
& $-0.0539 \cos \left(\lambda_{1}-5 \lambda_{2}-p_{1}-3 p_{2}\right)$ & $0.0213 \cos \left(\lambda_{1}-5 \lambda_{2}-2 p_{1}-2 p_{2}\right)$ \\
& $-0.0277 \cos \left(\lambda_{1}-5 \lambda_{2}-3 p_{1}-p_{2}\right)$ & $0.0129 \cos \left(\lambda_{1}-5 \lambda_{2}-p_{1}-3 p_{2}\right)$ \\
& $-0.0199 \cos \left(\lambda_{1}-5 \lambda_{2}-2 p_{1}-2 p_{2}\right)$ & $-0.01117 \cos \left(\lambda_{2}+p_{2}\right)$ \\
\hline 0.341 & $0.206 \cos \left(\lambda_{1}-5 \lambda_{2}-2 p_{1}-2 p_{2}\right)$ & $-0.156 \cos \left(\lambda_{1}-5 \lambda_{2}-p_{1}-3 p_{2}\right)$ \\
& $-0.164 \cos \left(\lambda_{1}-5 \lambda_{2}-p_{1}-3 p_{2}\right)$ & $0.0652 \cos \left(\lambda_{1}-5 \lambda_{2}-2 p_{1}-2 p_{2}\right)$ \\
& $-0.0852 \cos \left(\lambda_{1}-5 \lambda_{2}-3 p_{1}-p_{2}\right)$ & $0.0392 \cos \left(\lambda_{1}-5 \lambda_{2}-p_{1}-3 p_{2}\right)$ \\
\hline
\end{tabular}
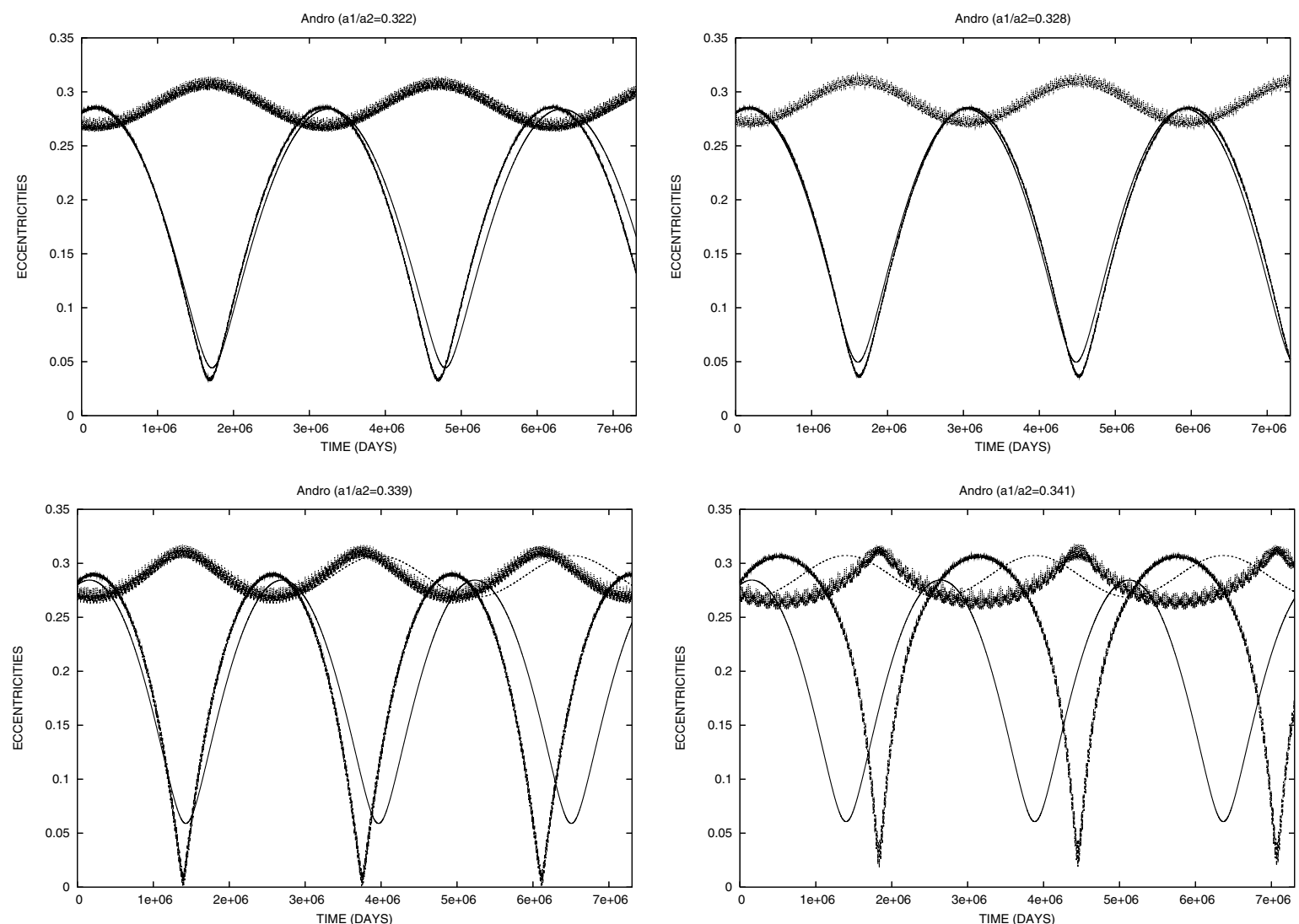

Fig. 1. Comparison between the time evolution of $e_{1}$ and $e_{2}$ as given by our analytical secular theory (solid line for $e_{1}$ and dashed line for $e_{2}$ ) and the numerical integration of the full three-body problem computed using SWIFT with the time of periastron passage of the outer planet as the initial epoch (curves including short-period variations). We take all initial conditions of $v$ Andromedae system except the outer semi-major axis for which we choose: $a_{2}=2.575$ (top left), $a_{2}=2.530$ (top right), $a_{2}=2.445$ (bottom left), $a_{2}=2.431$ (bottom right).

the main perturbing term is a non resonant one; also the $11 / 2$ resonance $(0.1818)$ is very close to the ratio of frequencies $(0.1846)$, but this resonance does not seem to affect the motion.

For $a_{1} / a_{2}=0.339$, the terms in " $\lambda_{1}-5 \lambda_{2}$ " are dominant. The motion is still described qualitatively by the secular theory but not quantitatively, as seen from Fig. 1. Finally, in the case of $a_{1} / a_{2}=0.341$, the system is inside the mean-motion resonance, so our approach fails completely.

As for comparing the time evolutions of the eccentricities, let us point out that the analytically averaged evolutions over time were computed on the basis of osculating initial conditions. It is expected that a better fit would be obtained by using initial conditions for averaged variables that could be computed from Sect. 2.

\section{Conclusion}

We have analysed the proximity of several exoplanetary systems to mean-motion resonances by using a first-order (in the mass ratios) Lie algorithm on the perturbative potential expanded in powers of the eccentricities. It has enabled us to find the limit of application of our secular analytical model for systems close to resonances. We have seen that the exosystems HD 168443, HD 38529, HD 74156, HD 217107, and HD 190360 are far away 
from a mean-motion resonance zone. In contrast, $v$ Andromedae, HD 12661, and HD 169830 systems are rather close to the 5/1 or the 9/1 resonance, but their behaviour can be adequately depicted by purely secular perturbation alone. Finally, the exosystems HD 108874 and HD 202206 suffer from large perturbations in their motion due to the closeness of the 4/1 and 5/1 resonances, respectively. Such resonant terms have to be taken into account in the long-term evolution of these exosystems.

Acknowledgements. We thank U. Locatelli for his constructive remarks in Celmec IV. We also would like to thank M. Duncan and H. Levison for the use of the SWIFT software package.

\section{References}

Beaugé, C., Michtchenko, T. A., \& Ferraz-Mello, S. 2006, MNRAS, 365, 1160 Callegari, N., Michtchenko, T. A., \& Ferraz-Mello, S. 2004, Celest. Mech. \& Dyn. Astro, 89, 201
Brouwer, D., \& Clemence, G. M. 1961, Methods of celestial mechanics (New York: Academic Press)

Chiang, E. I., Tabachnik, S., \& Tremaine, S. 2001, AJ, 122, 1607

Deprit, A. 1969, Celest. Mech., 1, 12

Henrard, J., \& Lemaître, A. 1987, Icarus, 69, 266

Hori, G.-I. 1966, PASJ, 18, 287

Ji, J.-H., Liu, L., Zhou, J.-L., \& Kinoshita, H. 2003, Chinese Astron. Astrophys., 27,127

Laskar, J. 1990, Systèmes de Variables et Eléments, in Les Méthodes modernes de la Mécanique Céleste, ed. D. Benest, \& C. Froeschlé (Éditions Frontières), 63

Lee, M. H., \& Peale, S. J. 2003, ApJ, 592, 1201

Lemaître, A., \& Henrard, J. 1988, Celest. Mech. \& Dyn. Astron., 43, 91

Libert, A.-S., \& Henrard, J. 2005, Celest. Mech. \& Dyn. Astron., 93, 187

Libert, A.-S., \& Henrard, J. 2006, Icarus, 183, 186

Michtchenko, T. A., \& Malhotra, R. 2004, Icarus, 168, 237

Michtchenko, T. A., \& Ferraz-Mallo, S. 2001, Icarus, 149, 357

Murray, C. D., \& Dermott, S. F. 1999, Solar System Dynamics (Cambridge University Press)

Psychoyos, D., \& Hadjidemetriou, J. 2005, Celest. Mech. \& Dyn. Astron., 92, 135

Rodríguez, A., \& Gallardo, T. 2005, ApJ, 628, 1006

Wisdom, J., \& Holman, M. 1991, AJ, 102, 1528 